\title{
Corrosion behavior of zirconia in acidulated phosphate fluoride
}

\author{
Anie THOMAS ${ }^{1}$, Sathyanarayanan SRIDHAR ${ }^{1}$, Shant AGHYARIAN ${ }^{1}$, Pilanda WATKINS-CURRY², Julia Y. CHAN², \\ Alessandro POZZI ${ }^{3}$, Danieli C. RODRIGUES
}

1- University of Texas at Dallas, Department of Biomedical Engineering, Richardson, USA.
2- University of Texas at Dallas, Department of Chemistry, Richardson, USA.
3- Polytechnic University of Marche, Department of Oral Surgery-Implant Dentistry, Ancona, Italy.

Corresponding address: Danieli C. Rodrigues - 800 W. Campbell Road - Richardson - Texas - 75080 - USA - Phone: 9728834703 - e-mail: danieli@utdallas.edu Submitted: September 16, 2015 - Modification: November 30, 2015 - Accepted: December 02, 2015

\section{ABSTRACT}

\begin{abstract}
bjective: The corrosion behavior of zirconia in acidulated phosphate fluoride (APF) representing acidic environments and fluoride treatments was studied. Material and Methods: Zirconia rods were immersed in $1.23 \%$ and $0.123 \%$ APF solutions and maintained at $37^{\circ} \mathrm{C}$ for determined periods of time. Surfaces of all specimens were imaged using digital microscopy and scanning electron microscopy (SEM). Sample mass and dimensions were measured for mass loss determination. Samples were characterized by powder X-ray diffraction (XRD) to detect changes in crystallinity. A biosensor based on electrochemical impedance spectroscopy (EIS) was used to detect ion dissolution of material into the immersion media. Results: Digital microscopy revealed diminishing luster of the materials and SEM showed increased superficial corrosion of zirconia submerged in $1.23 \%$ APF. Although no structural change was found, the absorption of salts (sodium phosphate) onto the surface of the materials bathed in $0.123 \%$ APF was significant. EIS indicated a greater change of impedance for the immersion solutions with increasing bathing time. Conclusion: Immersion of zirconia in APF solutions showed deterioration limited to the surface, not extending to the bulk of the material. Inferences on zirconia performance in acidic oral environment can be elucidated from the study.
\end{abstract}

Keywords: Dental abutments. Dental implants. Dental crowns. Acidulated phosphate fluoride. Corrosion. Ceramics.

\section{NTRODUCTI ON}

Zirconia and its alloys are gaining prevalence in the dental implant sector because of their aesthetic properties, biocompatibility, low plaque surface adhesion, high flexural strength, desirable osseointegration ${ }^{10}$, absence of mucosal discoloration ${ }^{12}$ and corrosion resistance ${ }^{4}$. The ongoing research for aesthetic, functionally stable and biocompatible materials has favored the use of all-ceramic reconstructions for fixed dental prostheses (FDPs) as alternatives to conventional porcelain-fused-to-metal (PFM) prostheses ${ }^{11}$. Highstrength metal-oxide ceramics have been developed to overcome the mechanical drawbacks and high fracture rates of previous all-ceramic systems ${ }^{23}$. Ceramics are effectively replacing the metallic components of prosthetic restorations ${ }^{16}$. Alumina was the first bioceramic fabricated to serve as part of dental prosthetics; however, its inherent low bending strength and fracture toughness lead to failure due to the fractures. With higher fracture resistance and elastic modulus, zirconia became the material of choice succeeding alumina. Yttria-stabilized zirconium dioxide (Y-TZP) is more biocompatible than its metal alternatives, preventing soft tissue inflammation and discoloration ${ }^{1,12}$. White and shaded zirconia frameworks prevent bluish discoloration of peri-implant soft tissues and may be beneficial if soft tissue recession occurs in the long term. Thus, Y-TZP contributes to achieving healthy soft tissue integration of implant-supported restorations, thus improving long-term stability of the marginal bone ${ }^{19}$. Zirconia-based all-ceramics are currently used to fabricate copings, implant abutments, partial and complete arch frameworks 
on both natural teeth and implants, in both anterior and posterior oral cavity areas ${ }^{13,17,19-21}$. The success rates of these frameworks are comparable with conventional PFM FDPs, but it is important to note that these statistics are reports from three to five years of clinical use ${ }^{15}$.

In spite of the reported success, clinical reports of zirconia's catastrophic failures do exist with significant biological and technical cost ${ }^{3}$. Generally, failures of ceramics are highly associated with fracture due to the inherent brittleness of the material ${ }^{12}$. Despite this, zirconia constitutes a superior fracture toughness stabilized by the transformation in the crystalline structure. However, there are certain environmental conditions that can lead to degradation of the material. One of the most important environmental degradation mechanisms associated with zirconia is known as Ageing (or) Low Temperature Degradation (LTD) ${ }^{14}$. The process of ageing takes place in two different scenarios: (i) stress corrosion: slow transformation of surface particles to the monoclinic phase over time; (ii) chemical degradation: a chemisorption of $\mathrm{OH}^{-}$from water at the surface of zirconia grains to form $\mathrm{Y}(\mathrm{OH})_{3}$ and depletion of Yttrium. Transformation occurs in both scenarios and is often ensued by a nucleation and growth process. This phenomena can lead to a cascade of events such as crack growth on the surface which is termed Subcritical Crack Growth (SCG) or static fatigue ${ }^{6,14,29}$. These processes can trigger in vivo release of ceramic particles. Generally, ceramic particles are very inert and do not trigger adverse reactions, however there have been studies pointing to the ability of zirconia powder to cause inflammatory reactions due to the formation of zirconium hydroxide ${ }^{16}$. Furthermore, investigation of the presence of ceramic particles in aspirated synovial fluid is considered as a possible diagnostic tool for early detection of fracture in ceramic-on-ceramic (COC) hip implants ${ }^{25}$.

However, the formation of zirconium hydroxide has now been overcome with the sintering process ${ }^{16}$. Nevertheless, the main clinical concern reported in the literature regarding Y-TZP used as a framework material is a higher incidence of veneering porcelain chip-off fracture rates ${ }^{10,11}$, which has been reported to range from $15 \%$ to $54 \%$ over a 3 - to 5 -year period ${ }^{11,12}$, versus $2.9 \%$ to $8.8 \%$ ceramic fracture rates observed in conventional tooth- and implant-supported metal-ceramic restorations over 5 years ${ }^{18}$. Despite this mechanical complication, patient satisfaction, favorable soft tissue response and high aesthetic outcome were noted ${ }^{18}$. Several hypotheses concerning the causes of porcelain veneer chipping highlighted the importance of factors such as framework design, laboratory handling, baking procedures and ceramic mechanical properties ${ }^{15}$.
Hence, it is important to investigate and understand the effect of unfavorable acidic aqueous environment on the surface of newly incorporated materials such as zirconia. It has been also suggested that an aqueous environment with acidic conditions can accelerate the process of ageing with crack growth decreasing 20\%-30\% the prosthesis' lifetime ${ }^{4}$. The oral environment is an aqueous electrochemical medium with high chances of $\mathrm{pH}$ fluctuations triggered by the presence of bacteria, ingestion of foods or presence of an inflammatory condition $^{26}$. In addition to this, topical oral hygiene mouthwashes prescribed to patients contain highly acidic media due to sodium fluoride and hydrochloric $\operatorname{acid}^{30}$. Acidulated Phosphate Fluoride (APF) is one such common topical mouthwash used to combat dental caries. The effect of corrosive environments has been assessed for different dental ceramics where the low $\mathrm{pH}$ of the medium attacked the surface leading to coarser surfaces ${ }^{24}$. Although the influences of different $\mathrm{pH}$ values on zirconia have been evaluated, effects of clinical solutions, such as APF, on the surface of zirconia have not been investigated 28 .

Henceforth, this study focuses on the investigation of the surface characteristics of zirconia samples exposed to acidulated phosphate fluoride solutions $(0.123 \%$ and $1.23 \%)$ for an extended time period of $11 \mathrm{~d}$. This study is based on the hypothesis that an acidic aqueous medium in the oral environment can accelerate the process of ceramic ageing. This process can generate nucleation and propagation of cracks, in which fractures will result in dissolution of ceramic particles in the oral environment. Microscopic techniques were performed to understand the morphological features of the surface. X-ray diffraction (XRD) was employed to evaluate the crystal structure of the ceramic material because the change in the crystal structure is considered an indicator of material degradation. An electrochemical sensor was used to detect the presence of ceramic particles dissolution in the APF medium in which the samples were immersed. The overall goal of this study is to provide information on the performance of zirconia materials under simulated acidic oral environment.

\section{MATERIAL AND METHODS}

\section{Material}

A non-porous zirconia ceramic rod was obtained from a vendor (McMaster-Carr; Atlanta, Georgia, USA; $97 \% \mathrm{ZrO}_{2}$, 3\% trace elements) with diameter of approximately $0.15 \mathrm{~cm}$ and $30 \mathrm{~cm}$ in length. Acidulated phosphate fluoride $(1.23 \%)$ (Pediagel, pH3.8, Indian Trail, North Carolina, USA) was used to prepare the immersion solutions. 


\section{Preparation of the zirconia rods}

Ten zirconia rod sections were cut to an approximate length of $2.54 \mathrm{~cm}$ each and the ends smoothed to touch and visually using 500-grit silica paper (Pace Technologies, 500-grit, Tucson, Arizona, USA). Before immersion, all the samples were immersed in acetone and sonicated for $5 \mathrm{~min}$.

\section{I mmersion setup and testing}

The cleaned zirconia rods were divided into four groups involving different immersion conditions as follows:

Group 1: a zirconia rod not subjected to any immersion was used as a control.

Group 2: a zirconia rod immersed in deionized (DI) water.

Group 3: four zirconia rods immersed in $1.23 \%$ APF medium with each rod immersed in an isolated medium contained in separate glass vials.

Group 4: four zirconia rods immersed in $0.123 \%$ APF medium in a similar manner to group 3 . $0.123 \%$ APF was prepared by $1: 10$ dilution of the commercial topical agent.

The sample bathed in DI water represented a neutral $\mathrm{pH}$ oral environment for the material. Different concentrations of APF solutions represented different acidic oral environment conditions. The test samples were stored in a $37^{\circ} \mathrm{C}$ room to simulate oral temperature. It has been mentioned in a previous study that $24 \mathrm{~h}$ of immersion would represent 1 year of exposure to APF solution for 4 min every day ${ }^{13}$. Hence, the immersion test was performed for $11 \mathrm{~d}$ to represent exposure of restoration materials to APF for 10-11 years.

Surface morphology of zirconia rods from all the groups were analyzed at specific time intervals ( " $\mathrm{t}$ ") ( $\mathrm{t}=24$ h, 120 h, 192 h, 264 h). In addition, aliquots of immersion media from groups 2-4 were collected during the same time interval to verify the dissolution of zirconia particles release over prolonged exposure to acidic aqueous environment.

\section{Mass loss experiments}

Mass loss and change in dimension were recorded to verify if dissolution of zirconia ions/particles could occur under the experimental conditions. Weight and dimensions (length and width) were recorded periodically for $11 \mathrm{~d}$ (264 h) for all specimens using a microbalance and caliper, respectively. Weight loss percentage was calculated using Equation 1. Where $\% \Delta \mathrm{W}$ represents the percentage of weight loss; the initial weight of each specimen, before immersion, is denoted by $\mathrm{W}_{\mathrm{o}}$; and the weight of immersed zirconia rods every $24 \mathrm{~h}$ is indicated by $\mathrm{W}_{\mathrm{T}}$.

$$
\% \Delta W=\left(\frac{W_{0}-W_{T}}{W_{0}}\right) * 100
$$

\section{Surface morphology}

The effect of APF solutions on the material surface was analyzed using different microscopy techniques. Surfaces of all specimens were imaged using digital microscopy (Keyence VHX - 2000, Itasca, Illinois, USA), and analyzed by Scanning Electron Microscopy (SEM) (SEM, JEOL, JSM-6010, Peabody, Massachusetts, USA), before and after immersion.

\section{X-ray diffraction (XRD)}

Zirconia samples were ground using an alumina mortar and pestle. Powder X-ray diffraction (Bruker D8 Advance Powder Diffractometer, Madison, Wisconsin, USA) was used to determine the structure of the material pre- and post-immersion. Diffraction data were collected for a 2-theta range from 10 to 80 at 0.1 step size on a powder diffractometer equipped with a LYNXEYE detector with a power of $40 \mathrm{kV}$ and $40 \mathrm{~mA}$.

\section{Zirconia release study}

The release of zirconia particles due to surface degradation in the immersion solution was analyzed with an electrochemical biosensor chip platform that quantifies particle dissolution using Electrochemical Impedance Spectroscopy (EIS). Aliquots of testing solutions were collected before and after the immersion of zirconia rods. After the immersion of rods, sample aliquots were collected from the testing media at different time intervals " $\mathrm{t}$ " hours (h) $(\mathrm{t}=0,24 \mathrm{~h}, 120 \mathrm{~h}, 192 \mathrm{~h}, 264 \mathrm{~h})$.

The EIS test was performed at a constant peakto-peak voltage of $10 \mathrm{mV}$ and a frequency of 250 $\mathrm{Hz}$. The impedance of the sample of interest varies according to the conductivity of it, which depends on the concentration of ceramic particles in the electrolyte (i.e., a decrease in impedance indicates a more conductive medium). Deionized (DI) water which was not subjected to any immersion was used as the control baseline. The ratio of change in impedance of the sample medium analyte from baseline was calculated to generate a percentage change in impedance $(\Delta Z)$. Percent change for impedance was determined using Equation (2). This allowed the data set to be averaged and the standard deviation to be determined over three runs on three separate EIS chips for the $0.123 \%$ APF solution. Only one EIS measurement (one run per chip) was taken for the $1.23 \%$ APF gel due to the high viscosity of the medium. Where $\% \Delta Z$ represents the percent change in impedance; $Z_{c}$ and $Z_{\text {, }}$ represent the impedance value of the immersion solution before and after immersion of specimens, respectively.

$$
\% \Delta Z=\left(\frac{Z_{c}-Z_{I}}{Z_{c}}\right) * 100
$$




\section{RESULTS}

\section{Mass loss analysis}

Weight and dimension measurements of immersed zirconia samples showed only negligible change in both testing solutions. The average percentage of weight loss for samples immersed in $1.23 \%$ APF was about $-0.01 \%$, while samples immersed in $0.123 \%$ APF showed a weight loss of $0.01 \%$. The dimensions of the specimens showed similar characteristics, where there was no change compared to pre-immersion values.

\section{Surface morphology}

Digital microscopy images showed discoloration and loss of luster on the surface for the specimens immersed in APF solutions, as illustrated in Figure 1 and 2, when compared to the controls (Figure 1: 1A and Figure 2:10A). Zirconia immersed in DI water showed similar luster to that of the control. Manufacturing marks were visible on all surfaces of the zirconia rods whether they were immersed or dry as seen in Figure 1: $1 \mathrm{~A}-5 \mathrm{~A}$ and Figure 2:6A-10A.

SEM showed a smooth undisrupted surface for the control specimen (Figure 1:1B). The grain boundaries were visible and indicated a uniform

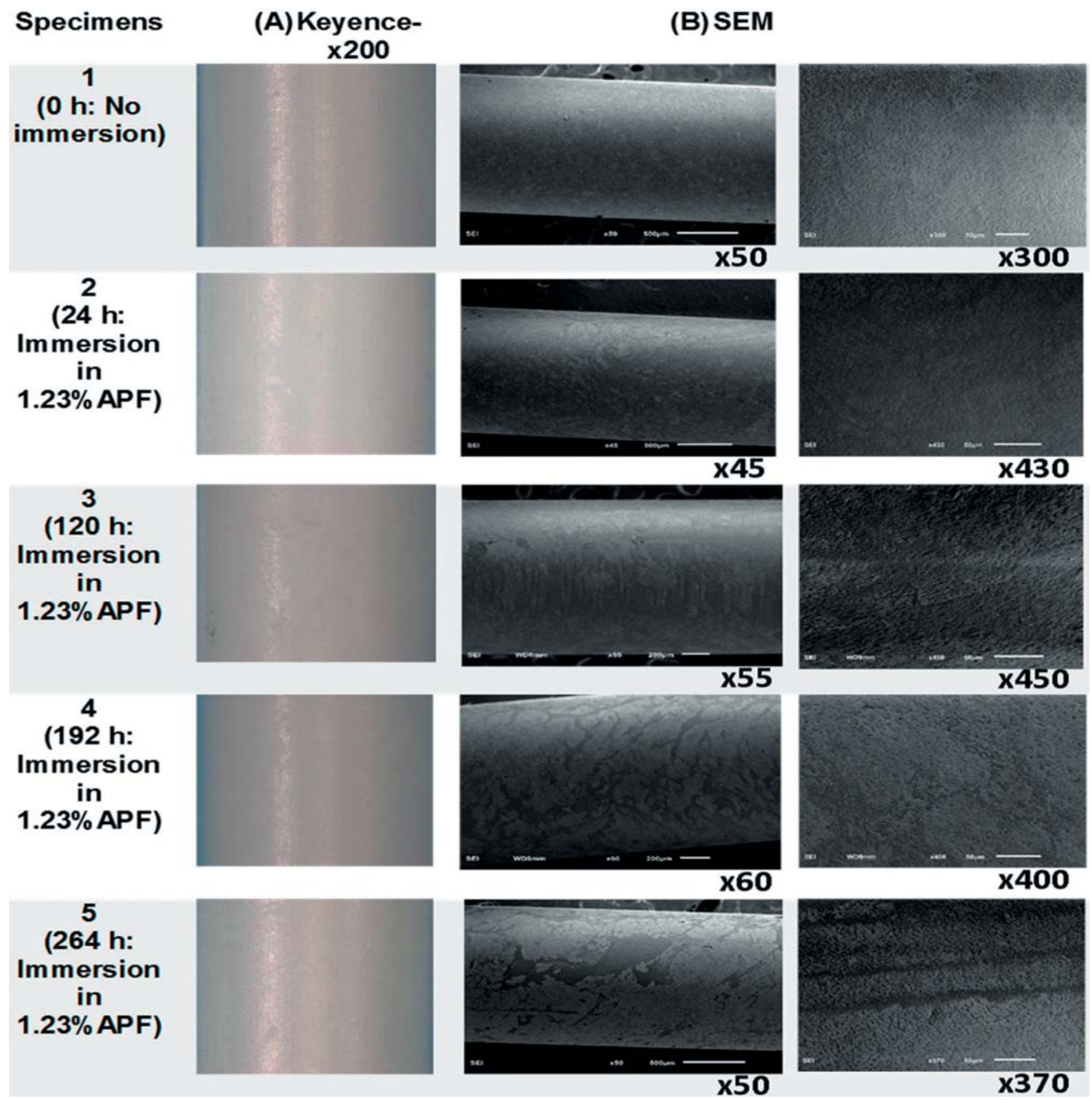

Figure 1- (A) Microscopic images at 200x magnification of zirconia surface before and after immersion in $1.23 \%$ Acidulated Phosphate Fluoride (APF) at $37^{\circ} \mathrm{C}$ for 24 h, 120 h, $192 \mathrm{~h}$, and $264 \mathrm{~h}$; (B) Scanning electron microscopy images of all zirconia specimens at different immersion times and solutions 
top layer. With immersion in APF solutions, the specimens showed imperfections on the superficial layers. The disruption of surface grain and formation of micro pores increased with immersion time in both $1.23 \%$ and $0.123 \%$ APF solutions, as illustrated in Figure 1:2B-5B and Figure 2:6B-9B. Zirconia bathed in DI water was comparable to the control where an intact top layer was evident (Figure 2: 10B).

\section{X-ray diffraction (XRD)}

XRD patterns for the control before immersion in DI water or APF solutions showed that zirconia was of the tetragonal phase as indicated in Figures
$3 a$ and $4 a$. This XRD pattern was used as a baseline for comparison to all the other specimens. For zirconia immersed in APF solutions, reflections corresponding to sodium phosphate $\left(\mathrm{Na}_{3} \mathrm{PO}_{4}\right)$ are evident and indicated by an asterisk $(*)$. As the immersion time increased, so did the intensities indicating the presence of higher concentration of sodium phosphate. Additionally, these peaks are more intense for the samples bathed in $0.123 \%$ APF solution (Figure 4) than in $1.23 \%$ APF solution (Figure 3). XRD patterns for the zirconia specimen in $\mathrm{DI}$ water showed peaks for sodium phosphate dehydrate $\left(\mathrm{Na}_{2} \mathrm{HPO}_{4} \cdot 2 \mathrm{H}_{2} \mathrm{O}\right)$ (Figure $4 \mathrm{f}$ ).

\section{Specimens \\ 6 $(24 \mathrm{~h}:$ Immersion in \\ $0.123 \%$ APF)}

(A) Keyence$\mathbf{x 2 0 0}$
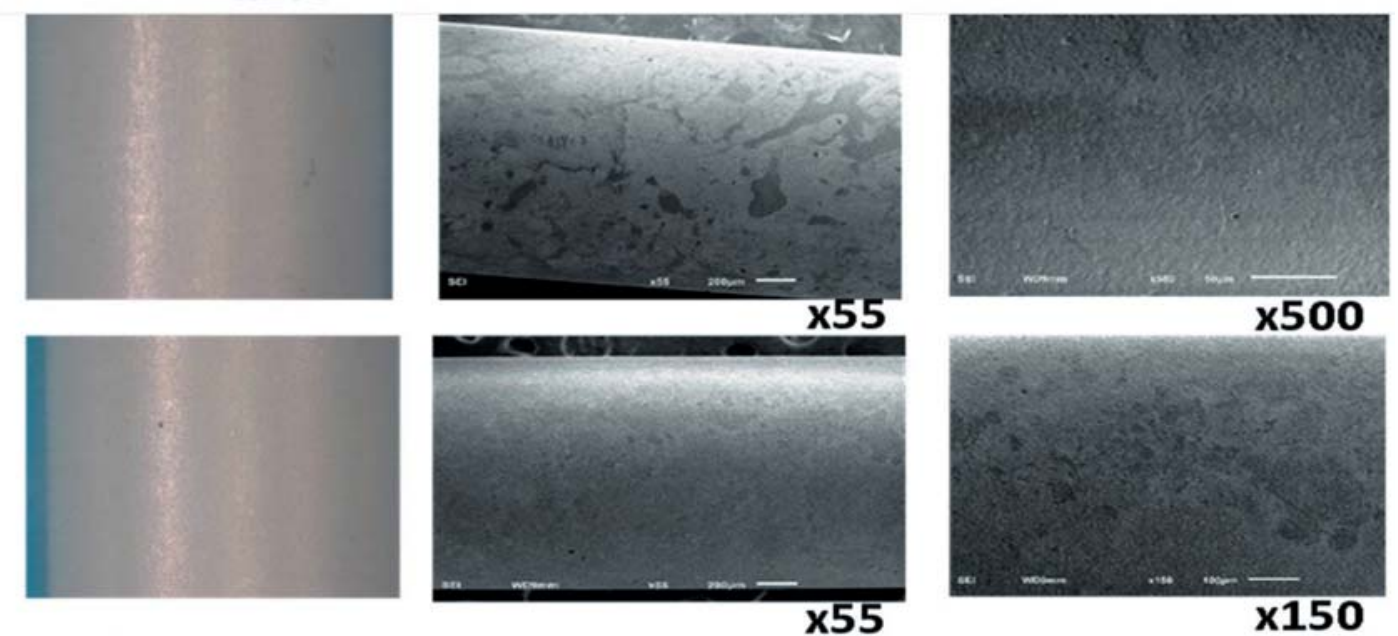

\section{8 \\ (192 h: \\ Immersion \\ in \\ $0.123 \%$ APF)}
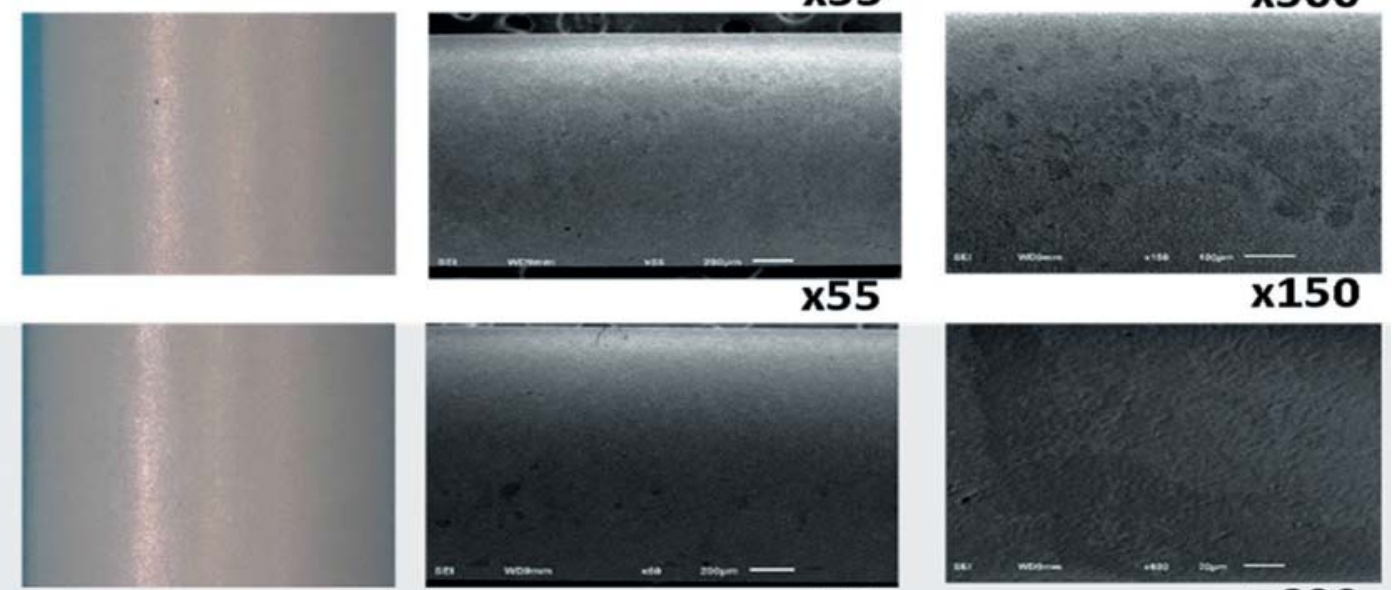

9

(264 h:

Immersion

in

$0.123 \%$ APF)
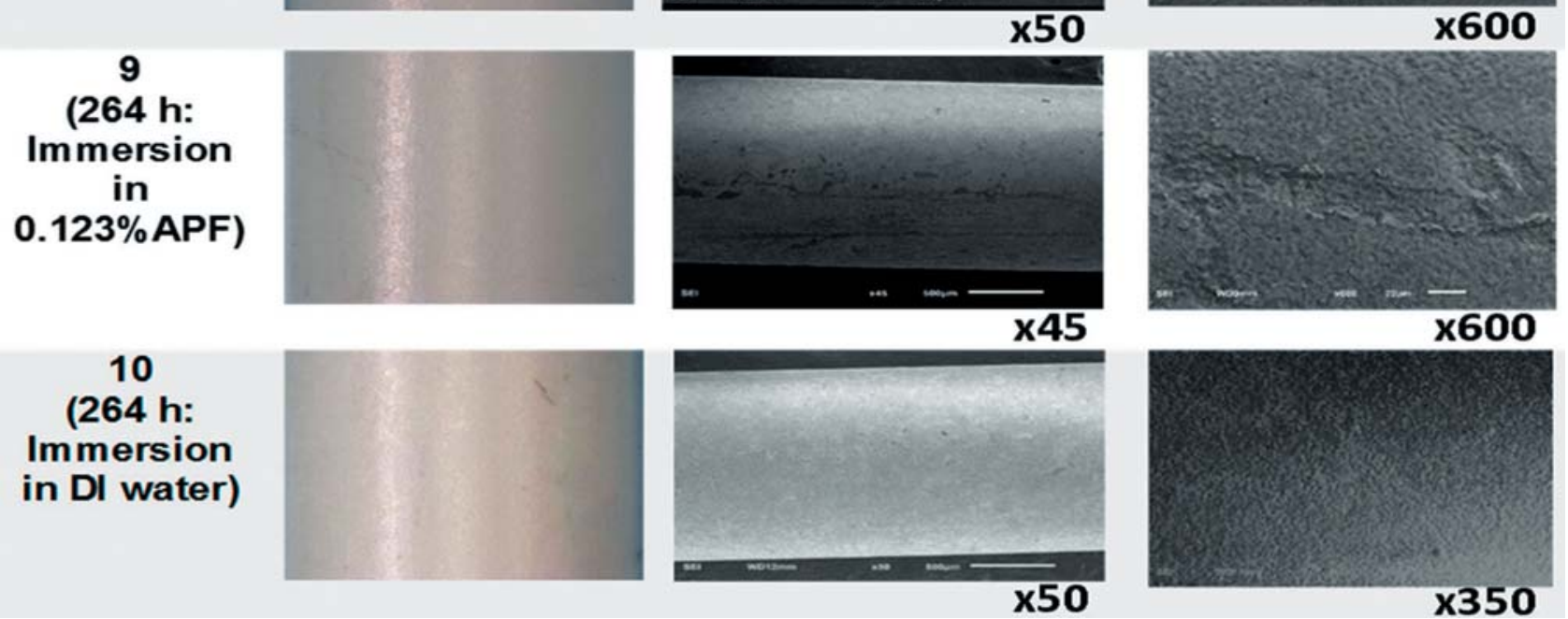

Figure 2- (A) Microscopic images at 200x magnification of zirconia surface after immersion in $0.123 \%$ Acidulated Phosphate Fluoride (APF) and deionized (DI) water at $37^{\circ} \mathrm{C}$ for 24 h, 120 h, 192 h, and 264 h; (B) Scanning electron microscopy images of all zirconia specimens at different immersion times and solutions 


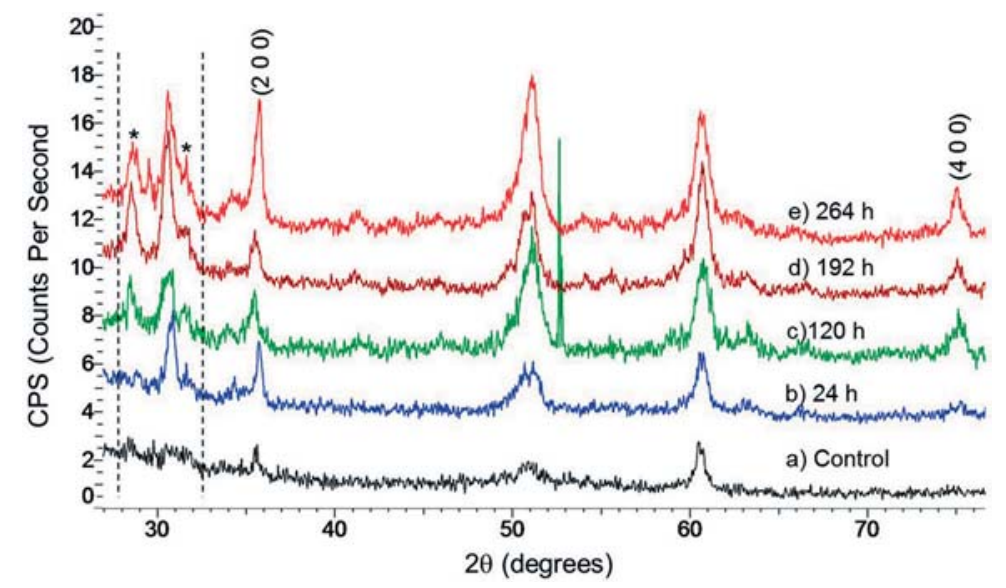

Figure 3- Powder diffraction patterns as a function of increasing immersion times in $1.23 \%$ Acidulated Phosphate Fluoride (APF) solution at oral temperature. (a) control (b) $24 \mathrm{~h}$ (c) $120 \mathrm{~h}$ (d) $192 \mathrm{~h}$ (e) $264 \mathrm{~h}$, * indicates $\mathrm{NaPO}_{3}$

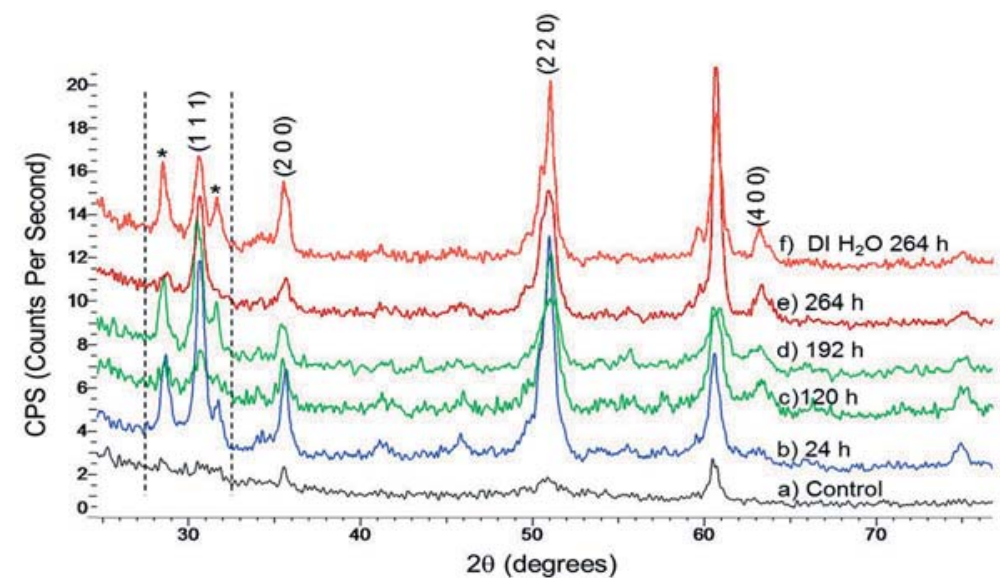

Figure 4- Powder diffraction patterns as a function of increasing soaking times in $0.123 \%$ Acidulated Phosphate Fluoride (APF) solution and deionized (DI) water at oral temperature. (a) control (b) $24 \mathrm{~h}$ (c) $120 \mathrm{~h}$ (d) $192 \mathrm{~h}$ (e) $264 \mathrm{~h}$ (f) DI water $264 \mathrm{~h},{ }^{*}$ indicates $\mathrm{NaPO}_{3}$

A

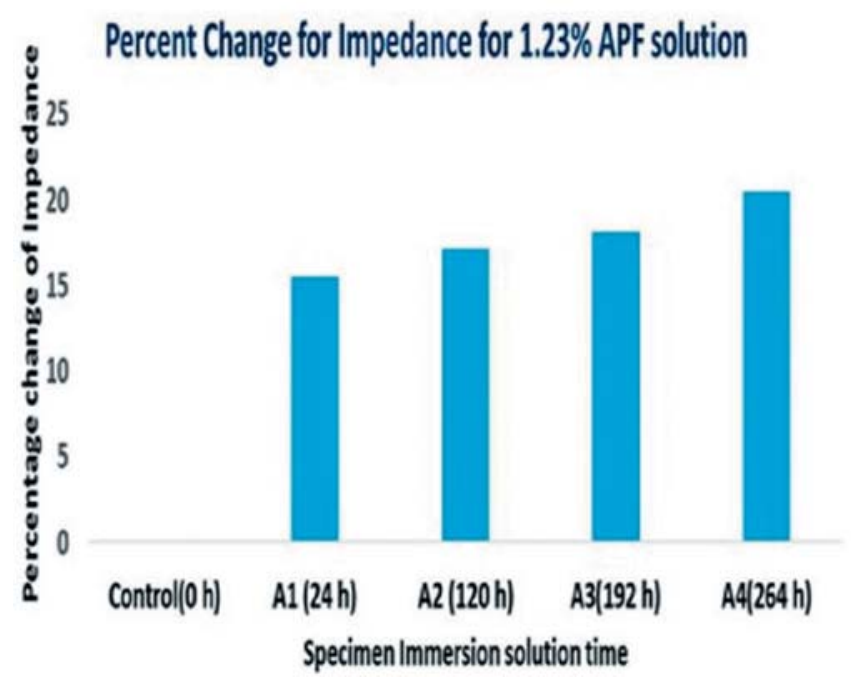

B

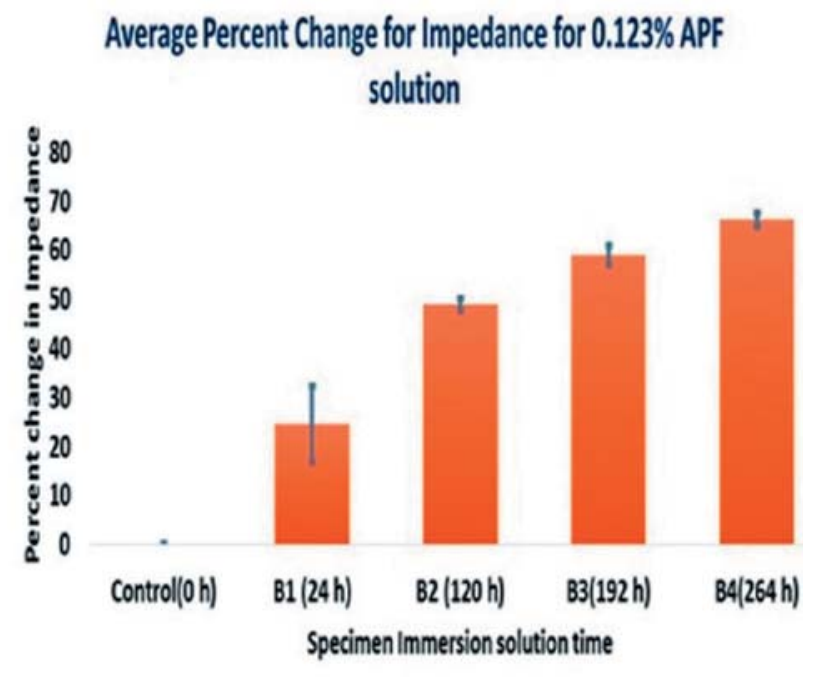

Figure 5- Electrochemical Impedance Spectroscopy- (A) Percent change in impedance for 1.23\% Acidulated Phosphate Fluoride (APF) solution and (B) Average percent change for $0.123 \%$ APF solution, over $0 \mathrm{~h}, 24 \mathrm{~h}, 120 \mathrm{~h}, 192 \mathrm{~h}$, and $264 \mathrm{~h}$ 
Electrochemical I mpedance Spectroscopy (EIS)-based biosensor

Sample aliquots of immersion media such as DI water, $1.23 \%$ and $0.123 \%$ APF over the specified intervals of time $(0 \mathrm{~h}, 24 \mathrm{~h}, 120 \mathrm{~h}, 172$ h, $264 \mathrm{~h}$ ) were analyzed using a biosensor chip platform. Impedance values obtained from the biosensor showed a decrease in impedance, with increasing immersion time of samples in APF, as shown in Figure 5A-B. This trend was consistent in the analysis of sample aliquots from $0.123 \%$ and $1.23 \%$ APF solution. This represents an increase in solution conductivity, which was evident with the increase in the percentage change in impedance $\% \Delta Z$. Its average increased by $20 \%$ in $1.23 \%$ APF and $65 \%$ in $0.123 \%$ APF media collected at $\mathrm{t}=264$ $\mathrm{h}$ in comparison to the control solution before immersion. EIS measurements were acquired with three different chips for $0.123 \%$ APF solution and averaged (Figure 5B). Measurements were conducted only once for the $1.23 \%$ APF gel (Figure $5 A)$.

\section{DISCUSSION}

The immersion study of zirconia rods in APF was performed to investigate the effect of an acidic electrochemical environment on the surface of zirconia. It was hypothesized that acidic aqueous environment provided by the topical agent can degrade the zirconia surface and accelerate the process of ageing, leading to micro-crack nucleation and propagation.

Examination of surface morphology of samples exposed to APF containing media showed visible discoloration under digital microscope (Figures 1 and 2). This observation was attributed to a grain structural change in the superficial layer, which may have affected light reflection on the surface of the material. Manufacturing marks evident on all specimens were considered to be manufacturing defects, since they were visible on the non-immersed control as well as shown in Figure 1: 1A-5A; Figure 2:6A-10A. Some of these deposits were etched out by APF from the specimens, leaving spots of black streaks (Figure 1:3A, Figure 2:6A and 9A. These superficial defects were evident in the SEM images, where the top layer seemed to be etched with apparent micro-pore formation (Figure 1:2B-5B; Figure 2:6B-9B). Similar disruption of the superficial layer and formation of micro-pores have been shown in studies where hot acidic surface treatment was applied on zirconia ${ }^{5}$. Previous literature studies suggest creation of microcracks in ceramic materials when exposed to ageing solutions ${ }^{14}$. This would further lead to several complications such as: (i) increase in surface roughness, which could increase plaque adhesion ${ }^{22}$, (ii) abrasion of antagonistic teeth ${ }^{2}$, and (iii) color change leading to improper aesthetic qualities of restorations ${ }^{12}$. APF at different concentrations has been reported to etch dental composites, porcelain, amalgam and dental cements in vitro ${ }^{7,9}$. Ageing is characterized by the propagation of deformation from the surface to the bulk. It was essential to investigate the bulk crystal structure of zirconia samples to verify the extent of acidic attack.

XRD was performed to verify the crystal structure transformation. But, changes in lattice structure were not detected by XRD with the samples investigated. Further XRD analysis showed the absorption of sodium phosphate onto the surface of zirconia, but weight change was not evident as mentioned in the mass loss analysis. The disruption of the top layer and the absorption of sodium phosphate is a dynamic process in solution; therefore, change in mass was not measurable. The corrosion effect was limited to the superficial layers of the material and can be considered to be of nano-thickness. Samples immersed in $0.123 \%$ APF had a higher concentration of sodium phosphate with increasing time, as demonstrated by the increased intensities of $\mathrm{Na}_{3} \mathrm{PO}_{4}$ reflections in the XRD diffraction pattern (Figure $4 \mathrm{~A}-\mathrm{E}$ ). This is because the diluted APF solution allowed the diffusion of ions to occur more freely unlike the $1.23 \%$ APF gel, whose high viscosity imposed a diffusion barrier for the movement of ions. Peaks of sodium phosphate hydrate seen for zirconia immersed in DI water (Figure 4F) were possibly a result of cross contamination of the APF immersion media caused by instruments used to extract the samples from the solutions.

Even though there was no change in the crystal lattice structure, both microscopic evidence and XRD results illustrated superficial surface degradation. Therefore, it was necessary to confirm the dissolution of zirconia particles in the immersion media. Sample aliquots of immersion media collected over specific interval of times were analyzed by an electrochemical biosensor chip platform. Electrochemical biosensor results showed more conductivity of the solution with increasing time, as illustrated in Figure 5(A-B). Correlations between EIS and XRD results showed the absorption of sodium phosphate onto zirconia for both concentrations of APF solutions. This phenomenon can be attributed to selective ion exchange of sodium phosphate from APF attaching to the surface of zirconia, leaving fluoride ions in the solution to allow for the formation of hydrofluoric acid $(\mathrm{HF})^{4}$. Commercial APF $(1.23 \%)$ has a fluoride content of 12,300 parts per million (ppm) with $0.34 \%$ of $\mathrm{HF}$, where its dilution to $0.123 \% \mathrm{APF}$ will provide a fluoride content of $1,230 \mathrm{ppm}^{8,30}$. In addition, the ion exchange phenomenon leading 
to the formation of HF can further exacerbate the medium. HF present in the APF solution has been associated with the dissolution of silica in the dental bioglass ceramics. However, the lack of silica phase in zirconia has been attributed to its improved resistance to acid etching. But, a recent study suggested the possibility of etching of zirconia due to $\mathrm{HF}$ with an increased exposure time ${ }^{24}$. Earlier studies have disproved the theory of dissolution of zirconia or yttria into water and, similarly, showed no weight change in aged specimens ${ }^{14,27}$. Considering that our ageing solution was acidic, and that SEM images revealed disruption of a superficial layer, dissolution of this nano-layer is assumed to have occurred, which resulted in an increase in the conductivity of the APF solutions. This dissolution of ions was more prevalent for the $0.123 \%$ APF medium, which was attributed to the viscosity of the solution. The $1.23 \%$ APF gel was viscous, slowing the movement of ions and interaction between the gel and surface of material, while the diluted APF allowed for chemical exchanges between the surface and solution to occur more freely.

The results of this study clearly showed that the aqueous acidic environment was able to trigger superficial surface degradation with the dissolution of ceramic particles limited to the surface and did not affect the bulk crystal structure as confirmed by XRD, which was considered as an indicator for ageing (or) slow micro-crack propagation.

The degradatation limited to the surface without affecting the bulk crystal structure could explain the clinical finding of no zirconia framework fractures experienced in well conducted clinical studies, resulting in overall prosthetic survival rates of $100 \%$ up to 5 years ${ }^{13,17,18}$. Thus, the ongoing development of new clinical and laboratory procedures have yielded successful results of the PFZ as a viable restorative alternative to $\mathrm{PFM}$, resulting in high patient satisfaction ${ }^{19}$.

However, superficial surface degradation should not be neglected. Due to the roughness induced by fluoride and acidic environments, the top layer could promote plaque retention creating a stage for peri-implant diseases to arise ${ }^{22}$. In Dentistry, zirconia is gaining importance as the material of choice for making abutments and crowns. Pozzi, et al. ${ }^{19}$ (2015), reported on zirconium oxide's high biocompatibility and low plaque surface adhesion contributing to successful soft tissue integration, assessing a extremely low cumulative plaque score with $93 \%$ of patients without gingivitis throughout follow-up, and the remaining $2 \%$ and $5 \%$ with respective mild and moderate gingivitis ${ }^{19}$. Viceversa, non-hygienic inaccessible restorations and roughed or not properly polished surfaces are significantly associated with implant loss and a high rate of peri-implantitis ${ }^{22}$.
It has also been suggested that formation of surface corrosion layers may influence the degree of degradation in addition to the effect of microstructure and environmental conditions ${ }^{4}$. Moreover, it has been reported that zirconia's strength is influenced by different surface treatments that produce different degrees and types of surface damage . $^{5}$.

Certain limitations exist within this study. This methodology provides an overview on the corrosive behavior of APF on zirconia in oral temperature. Nevertheless, in-vivo oral environment can have fluctuations in $\mathrm{pH}$, mastication forces, and other factors, that may further influence the materials' properties and performance.

\section{CONCLUSI ON}

This study investigated the effect of acidic aqueous electrochemical environment due to a topical solution. However, the oral environment is comprised of complex electrochemical factors such as saliva, enzymes, a wide variety of bacterial species, along with fluctuations in the $\mathrm{pH}$ and temperature conditions due to food particles and beverages. In addition, dental restoration materials in vivo are also exposed to mechanical factors such as occlusal forces due to mastication. It has been clearly stated that the mechanical properties of zirconia are affected under prolonged exposure in an aqueous medium. In the limited clinical evidences obtained so far, failure of zirconia has been primarily associated with fracture. Hence, future studies will include mechanical loading of zirconia in simulated oral environment to understand the interplay of mechanical and electrochemical factors that can accelerate degradation and fracture of the material.

I mmersed samples did not show any transformation of crystalline phase from monoclinic to tetragonal. Hence, it was concluded that the acidic medium was not able to trigger the ageing phenomenon. However, microscopic images detected superficial surface degradation. In the oral environment, superficial surface damage, due to acidic medium, can be further invigorated by cyclic occlusal forces which can have a direct effect on the bulk properties of the material. Future studies will focus on the synergistic effect of mechanical forces and acidic electrochemical environment, which may accelerate crack propagation resulting in fracture and failure of the zirconia core or chipping of the porcelain veneering layer.

\section{ACKNOWLEDGMENTS}

The authors thank Texas A\&M University Baylor College of Dentistry for granting use of the SEM facilities. We also thank Dr. Shalini Prasad, principal investigator of Biomedical Microdevices 
and Nanotechnology Laboratory for conducting the electrochemical sensor testing. The authors would like to acknowledge Dr. Thomas G. Wilson $\mathrm{J} r$., a private practitioner for providing us with the clinical topical agent.

\section{REFERENCES}

1- Abrahamsson I, Berglundh T, Glantz PO, Lindhe J. The mucosal attachment at different abutments. An experimental study in dogs. J Clin Periodontol. 1998;25:721-7.

2- Al-Hiyasat AS, Saunders WP, Sharkey SW, Smith GM, Gilmour WH. Investigation of human enamel wear against four dental ceramics and gold. J Dent. 1998;26:487-95.

3- Aramouni P, Zebouni E, Tashkandi E, Dib S, Salameh Z, Almas $\mathrm{K}$. Fracture resistance and failure location of zirconium and metallic implant abutments. J Contemp Dent Pract. 2008; 9:41-8.

4- Bergmann CP, Stumpf A. Dental ceramics: microstructure, properties and degradation, In: Bergmann $\mathrm{CP}$, eds. Topics in mining, metallurgy and materials engineering. Berlin: Springer; 2013. p. 67-84.

5- Casucci A, Mazzitelli C, Monticelli F, Toledano M, Osorio R, Osorio E, et al. Morphological analysis of three zirconium oxide ceramics: effect of surface treatments. Dent Mater. 2010;26:751-60.

6- Chevalier J. What future for zirconia as a biomaterial? Biomaterials. 2006; 27:535-43.

7- Darvell BW. The effect of topical fluoride on amalgam. Br Dent J. 1977; 142: 47-51.

8- Dental products [Internet]. New York: Fluoride Action Network.; c2015. [cited 2015 Aug 31]. Available from: http://fluoridealert. org/issues/dental-products/toothpastes/

9- El-Badrawy WA, McComb D, Wood RE. Effect of home-use fluoride gels on glass ionomer and composite restorations. Dent Mater. 1993; 9:63-7.

10- Guess PC, Att W, Strub JR. Zirconia in fixed implant prosthodontics. Clin Implant Dent Relat Res. 2012; 14:633-45.

11- Heintze SD, Rousson V. Survival of zirconia- and metalsupported fixed dental prostheses: a systematic review. Int J Prosthodont. 2010; 23:493-502.

12- Koutayas S, Vagkopoulou T, Pelekanos S, Koidis P, Strub JR. Zirconia in dentistry: part 2. Evidence-base clinical breakthrough. Eur J Esthet Dent. 2009; 4:348-80.

13- Larsson C, Vult von Steyern P, Nilner K. A prospective study of implant-supported full-arch yttria-stabilized tetragonal zirconia polycrystal mandibular fixed dental prostheses: three-year results. Int J Prosthodont. 2010;23:364-9.

14- Lawson S. Environmental degradation of zirconia ceramics. J Eur Ceram Soc. 1995; 15: 485-502.

15- Lima JM, Souza AC, Anami LC, Bottino MA, Melo RM, Souza RO. Effects of thickness, processing technique, and cooling rate protocol on the flexural strength of a bilayer ceramic system. Dent Mater. 2013; 29: 1063-72
16- Manicone PF, Rossi Iommetti P, Raffaelli L. An overview of zirconia ceramics: basic properties and clinical applications. J Dent. 2007; 35:819-26.

17- Papaspyridakos P, Lal K. Computer-assisted design/ computer-assisted manufacturing zirconia implant fixed complete prostheses: clinical results and technical complications up to 4 years of function. Clin Oral Implant Res. 2013;24:659-65.

18- Pjetursson BE, Brägger U, Lang NP, Zwahlen M. Comparison of survival and complication rates of tooth-supported fixed dental prostheses (FDPs) and implant-supported FDPs and single crowns (SCs). Clin Oral Implants Res. 2007; 18:97-113.

19- Pozzi A, Holst S, Fabbri G, Tallarico M. Clinical reliability of CAD/CAM cross-arch zirconia bridges on immediately loaded implants placed with computer-assisted/template-guided surgery: a retrospective study with a follow-up between 3 and 5 years. Clin Implant Dent Relat Res. 2015; 17: e86-e96.

20- Pozzi A, Tallarico M, Barlattani A. Monolithic lithium disilicate full-contour crowns bonded on CAD/CAM zirconia complete-arch implant bridges with 3 to 5 years of follow-up. J Oral Implantol. 2015; 41:450-8.

21- Pozzi A, Tallarico M, Moy PK. The implant biologic pontic designed interface: description of the technique and cone-beam computed tomography evaluation. Clin Implant Dent Relat Res. 2015; 17(Suppl 2): e711-20.

22- Quirynen M, De Soete M, van Steenberghe D. Infectious risks for oral implants: a review of the literature. Clin Oral Implants Res. 2002; 13: 1-19.

23- Sailer I, Feher A, Filser F, Gauckler LJ, Luthy H, Hammerle CH. Five-year clinical results of zirconia frameworks for posterior fixed partial dentures. Int J Prosthodont. 2007; 20: 151-6.

24- Sriamporn T, Thamrongananskul N, Busabok C, Poolthong $\mathrm{S}$, Uo M, Tagami J. Dental zirconia can be etched by hydrofluoric acid. Dent Mater J. 2014; 33:79-85.

25- Stea S, Traina F, Beraudi A, Montesi M, Bordini B, Squarzoni $S$, et al. Synovial fluid microanalysis allows early diagnosis of ceramic hip prosthesis damage. J Orthop Res. 2012;30: 1312-20. 26- Surdacka A, Strzykaa K, Rydzewska A. Changeability of oral cavity environment. Eur J Dent. 2007; 1: 14-7.

27- Tinschert J, Natt G, Mohrbotter N, Spiekermann H, Schulze $K A$. Lifetime of alumina- and zirconia ceramics used for crown and bridge restorations. I Biomed Mater Res B Appl Biomater. 2007; 80: 317-21.

28- Turp V, Tuncelli B, Sen D, Goller G. Evaluation of hardness and fracture toughness, coupled with microstructural analysis, of zirconia ceramics stored in environments with different $\mathrm{pH}$ values. Dent Mater J. 2012; 31:891-902.

29- Volpato CM, Garbelotto LG, Fredel MC, Bondioli F. Application of zirconia in dentistry: biological, mechanical and optical considerations. In: Sikalidis C, ed. Advances in ceramics - electric and magnetic ceramics, bioceramics, ceramics and environment. Rijeka: InTech; 2011. p. 397-420.

30- Whitford GM, Adair SM, Hanes CM, Perdue EC, Russell CM. Enamel uptake and patient exposure to fluoride: comparison of APF gel and foam. Pediatr Dent. 1995; 17: 199-203. 